\title{
Unterwegs-Sein, für immer
}

\author{
Auf den Spuren Jean Gebsers
}

Elmar Schübl

topographie für ein weites land

alles auf sehnsucht einrichten nichts auf gewohnheit das natürliche ist das chaos die seele ein fluss wenn er über die ufer bricht das wasser in den händen die erde unter den augen der himmel hat hinter den wolken eine blaue haut und wartet bis der baum aus dem nebel seine arme in der luft verzweigt der wind aus süden den tau von knospen liest die weite einen herzschlag näher kommt zu dir und du den horizont wie ein blatt papier in deine brusttasche faltest wo immer du auch hingehen willst ${ }^{1}$

Das Gedicht »topographie furr ein weites land « ist das erste aus Albert Ostermaiers Band Solarplexus (2004). Ich werde in Unterwegs-Sein, für immer den Horizont aus der Brusttasche ziehen und ihn wie ein Blatt Papier entfalten. Diese Entfaltung eröffnet Erfahrungsräume Sphären -, in denen Welt erst für uns da ist, und wo wir Welt auch deuten. Es gilt in diesem Text eine hermeneutische Topographie vorzustellen, der Text ist ein Stück weit auch ein hermeneutischer Reisebericht.

Die menschliche Grunderfahrung, dass wir immer schon in Räumen enthalten und darin unterwegs sind, hat Peter Sloterdijk in seiner Sphären-Trilogie $(1998,1999,2004)$ in einem umfassenden Sinn behandelt. Er spürt der Raumerfahrung nach, die sich als elementare Existenzerfahrung erweist. Es sind Raum- und Existenzerfahrungen sowie deren Übergänge als Grenzerfahrungen, die Sloterdijk be-

1 Albert Ostermaier, Solarplexus. Gedichte, Frankfurt a.M. 2004, 7. 
schäftigen. Wir loten Erfahrungsräume aus, die uns Schritt für Schritt vertrauter werden, in denen wir uns schließlich zuhause fühlen, um dann doch den Schritt hinaus in einen noch unvertrauten Raum zu setzen. Rüdiger Safranski hat dazu bemerkt:

"Es gehört zum Drama des Lebens, dass man beseelte Räume, in denen man enthalten ist, immer wieder verlassen muss, ohne zu wissen, ob man lebbaren Ersatz in neuen Räumen finden wird. ${ }^{2}$

Diese Grundproblematik hat eine persönliche und eine menschheitliche Dimension.

Wir sind immer schon unterwegs in Räumen der äußeren Welt und zugleich in Weltinnenräumen. Das Unterwegs-Sein zählt zu den anthropologischen Grundbestimmungen und ist eine fundamentale Seinsweise menschlichen Daseins. Wir sind Wandelnde, als solche Erfahrungsräume Erkundende und sich in Sphären heimisch Einrichtende, um uns schließlich doch in neuartigen Erfahrungsräumen wieder zu finden. Dort, im noch Ungeheuren, weil Unvertrauten, setzen wir die Reise fort. (Nicht selten getrieben von Sehnsucht, die zur Gewohnheit mutiert.)

\section{I - Eine Annäherung}

Dieser immerwährende Wandel wird von mir in den Blick genommen.Das geschieht aus der Perspektive des Denkens von Jean Gebser, ${ }^{3}$ der über die Dichtung und seine literarische Übersetzungstätigkeit zum Philosophieren fand. Er hat als Außenseiter in der akademischen Welt einen bedeutenden Beitrag zur Bewusstseinsforschung geleistet. Der Wandel des menschlichen Bewusstseins ist das große Thema seines zweibändigen Hauptwerks Ursprung und Gegenwart $(1949 / 53)$. Es ist ein vielschichtiges Werk, das in unterschiedlichen fachlichen Kontexten verortet werden kann.

Ursprung und Gegenwart ( $U \& G$ ) steht in meiner Einführung ${ }^{4}$ der Neuausgabe nicht zuletzt in einem hermeneutischen Kontext. Ich

\footnotetext{
2 Rüdiger Safranski, Selbstverhältnisse, Weltverhältnisse. In Sloterdijks Sphäre, in: Die Vermessung des Ungeheuren. Philosophie nach Peter Sloterdijk, hg. von Marc Jongen, Sjoerd van Tuinen und Koenraad Hemelsoet, München 2009, 73-79, hier 76.

3 Elmar Schübl, Jean Gebser (1905-1973). Ein Sucher und Forscher in den Grenzund Übergangsgebieten des menschlichen Wissens und Philosophierens, Zürich 2003. 4 Elmar Schübl, Jean Gebsers "Ursprung und Gegenwart « im kulturphilosophischen, geschichtsphilosophischen und hermeneutischen Kontext, in: Jean Gebser, Ursprung und Gegenwart, Erster Teil: Die Fundamente der aperspektivischen Welt. Beitrag zu einer Geschichte der Bewußtwerdung (JGR, 1), Zürich 2015, 23-60.
} 
habe dort an einen Gedanken Heideggers aus Sein und Zeit (1927) angeknüpft: Verstehendes Entwerfen vollzieht sich aus einer »VorStruktur" heraus. Heideggers Vor-Struktur, als hermeneutischer Zirkel, erweist sich bei Gebser als komplexes Strukturgefüge, das sich durch eine bewusstseinsgeschichtliche Dimension auszeichnet. Seine Konzeption der Bewusstseinsstrukturen, die das tragende Element von $U \& G$ ist, bietet eine hermeneutische Topographie des menschlichen Bewusstseins und Wegmarken des hermeneutischen Reiseberichts.

Fünf Verfassungen des Bewusstseins - die archaische, die magische, die mythische, die mentale und die integrale Struktur - bilden das Strukturgefüge. Das Archaische ist die Grundstruktur, die sich durch ein teleologisches Moment auszeichnet; aus ihr konstellieren sich (in der genannten Reihenfolge) das Magische, das Mythische, das Mentale und das Integrale. Diese vier Seinsweisen sind spezifische Ausdrucksformen und Dimensionen des Archaischen. Das Integrale fasst Gebser als die bewusste Form des Archaischen auf.

Menschen entwerfen aus strukturgebundenen Gestaltungsmöglichkeiten Erfahrungsräume, die sie ausgestalten, indem sie auf weitverzweigten Wegen in diesen Sphären unterwegs sind. Dieses Ausgestalten bedeutet nicht zuletzt, dass (vergangene wie gegenwärtige) Menschen in intersubjektiven Erfahrungsräumen einander bestätigen, was sie für real halten. Die Strukturgebundenheit des Bewusstseins setzt jeweils die Grenzen der Gültigkeitsansprüche. Es handelt sich um innere Wahrheiten des Auffassungen von Wirklichkeit realisierenden Subjekts, die stets Ausdruck strukturgebundener Möglichkeiten des Selbst- und Weltverständnisses des Menschen sind. Das Magische, das Mythische, das Mentale und das Integrale sind fundamentale Seinsweisen mit spezifischen, ihnen entsprechenden Erfahrungsräumen, und das von Gebser entworfene Strukturgefüge ist Ausdruck des hermeneutischen Zirkels im Sinne Heideggers.

Dieser betont:

"Das Entscheidende ist nicht, aus dem Zirkel heraus-, sondern in ihn nach der rechten Weise hineinzukommen. Dieser Zirkel des Verstehens ist nicht ein Kreis, in dem sich eine beliebige Erkenntnisart bewegt, sondern er ist der Ausdruck der existenzialen Vor-Struktur des Daseins selbst. Der Zirkel darf nicht zu einem vitiosum und sei es auch nur zu einem geduldeten herabgezogen werden. In ihm verbirgt sich eine positive Möglichkeit

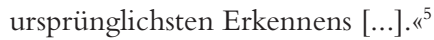

5 Martin Heidegger, Sein und Zeit, Tübingen ${ }^{18} 2001,153$. 
Die Bewusstseinsstrukturen eröffnen elementare Möglichkeiten menschlichen Erkennens. Gebsers Konzeption bietet mit dem Magischen, dem Mythischen, dem Mentalen und dem Integralen vier Einstiegsmöglichkeiten in den Zirkel des Verstehens. In Hinblick auf die Gestaltungsmöglichkeiten menschlichen Daseins bringen die archaische Grundstruktur und die integrale Bewusstseinsstruktur am deutlichsten die radikale Offenheit zum Ausdruck, die auch Heideggers hermeneutischen Ansatz in Sein und Zeit auszeichnet. Im integralen Erfahrungsraum der aperspektivischen Welt, der »Welt ohne Gegenüber «" die durch prinzipielle Offenheit charakterisiert ist, wird der Mensch zum "Navigator im total Offenen ${ }^{7}$ (Bruno von Flüe).

Bewusstsein bedeutet bei Gebser grundsätzlich etwas Offenes, im Sinne eines schöpferischen Möglichseins. »Im Schöpferischen ist der Ursprung Gegenwart. $\ll^{8}$

Bewusstsein, als schöpferisches Möglichsein, ist etwas Offenes, auch im Sinne von auf Wandlung Angelegtem. Dieser Wandel bedeutet jeweils einen Zuwachs an Reflexivität bzw. deren Intensivierung. Der Mensch ist im Magischen durch Ich-Losigkeit, im Mythischen durch ein Wir-Bewusstsein, im Mentalen durch ein Ich-Bewusstsein und im Integralen durch Ich-Freiheit bestimmt. Der Bewusstwerdungsprozess eröffnet dem Menschen stets neuartige Möglichkeiten des Selbst- und Weltbezugs und weist grundsätzlich ins Offene.

\section{II - Die Bewegung}

Jean Gebser hat den ersten Teil von $U$ \& $G$ als "Beitrag zu einer Geschichte der Bewusstwerdung "verfasst. Es ist ein Integrationsprozess, den er beschreibt. Die Bewusstwerdung ist Wandlung, deren Bewegung neuartige Erfahrungsräume eröffnet. Es handelt sich um eine Art Pendelbewegung zwischen den Polen der Selbst- und der Weltbezogenheit des Bewusstseins. Im Magischen und im Mentalen ist die Weltbezogenheit betont, im Mythischen und im Integralen ist es die Selbstbezogenheit.

\footnotetext{
${ }^{6}$ Jean Gebser, Ursprung und Gegenwart, Zweiter Teil: Die Manifestationen der aperspektivischen Welt. Versuch einer Konkretion des Geistigen (JGR, 1), Zürich 2015,671 .

7 Das Zitat stammt aus dem bislang unveröffentlichten Vortrag Gestaltordnungen der Seele, den Bruno von Flüe am 6. Juni 2014 im Internationalen Seminar für Analytische Psychologie (ISAP) in Zürich gehalten hat.

8 Gebser, Ursprung und Gegenwart, Zweiter Teil, 481.
} 
Gebser hebt außerdem das Spontane und Sprunghafte der Bewusstwerdung hervor. Sie erweist sich als "Mutationsgeschehen", aufgefasst als ein

"über und durch die Zeiten und Kulturen ausgeteiltes Sichtbarwerden anlagemäßig vorgegebener Bewußtseinsmöglichkeiten, welche teils mindernd, teils bereichernd, die jeweilige Wirklichkeitserfassung des Menschen bestimmen «?

Die Wandlung des Bewusstseins folgt aber nicht dem klassischen Entwicklungs- bzw. Fortschrittsgedanken. Das verdeutlicht Gebsers Unterscheidung zwischen effizienten und defizienten Formen des Bewusstseins. Von effizienten Ausdrucksformen kann gesprochen werden, wenn sie sich bei der Ausgestaltung strukturgebundener Möglichkeiten als fruchtbar erweisen. Das Effiziente bleibt in den verschiedenen Sphären geborgen, und mit jeder erschlossenen Struktur wird der entsprechende Erfahrungsraum komplexer.

Stellen effiziente Ausdrucksformen des Bewusstseins eine Bereicherung der Wirklichkeitserfassung des Menschen dar, so mindern defiziente Formen diese; sie werden gewissermaßen zum Dieb an der Wirklichkeit. ${ }^{10}$ Das Defiziente ist durch Erschöpfung charakterisiert, aus der sich keine effiziente (neuartige) Form bilden lässt.

Aus der Erfahrung des Integralen erweist sich der Bewusstwerdungsprozess als Nachvollzug dessen, was in der prinzipiellen Offenheit des schöpferischen Möglichseins im Archaischen bereits angelegt ist. In der Offenheit des Integralen schließt sich in gewisser Hinsicht ein Kreis. Das ist das zyklische Moment der von Gebser beschriebenen Entfaltung des Bewusstseins. Und das lineare Moment ist im Folgecharakter der Strukturen gegeben. Die Verlaufsform des Geschehens erweist sich so als spiralförmig, deren sinnstiftende Ordnungen als Bedeutungszusammenhänge in sich wandelnden Erfahrungsräumen zum Ausdruck kommen. Gebsers Strukturmodell beschreibt eine mehrdimensionale Wirklichkeit.

Im Verlauf der Menschheitsgeschichte haben sich immer mehr verbindliche Erfahrungsräume der einzelnen Bewusstseinsstrukturen ausgebildet. Sie können einander ergänzen, sich (teilweise)

9 Gebser, Ursprung und Gegenwart, Erster Teil, 115.

${ }^{10}$ Aus Gebsers Sicht bedeutet die auf reduktionistischen Verfahren basierende "Entzauberung der Welt" (Max Weber) die Eliminierung magischer und mythischer Momente in mentalen Erfahrungsräumen. Das Defiziente des Mentalen zeige sich nicht zuletzt in einer verengten rationalistischen Blickweise, durch die man des größeren Bezugs verlustig geht. Es zeige sich außerdem in der Auffassung, dass nur dasjenige Gültigkeit besitzt bzw. einen Wirklichkeitsanspruch erheben kann, das sich quantitativ erfassen lässt. 
durchdringen oder in Konkurrenz zueinander stehen. Im Prinzip können heute praktisch jederzeit sowohl effiziente als auch defiziente Formen sämtlicher Strukturen (beinahe) überall zum Ausdruck kommen. Wir sind temporär in magischen, mythischen und mentalen, aber auch schon in integralen Erfahrungsräumen unterwegs.

Die Seinsweisen des Magischen, des Mythischen, des Mentalen und des Integralen zeichnen sich durch spezifische Weisen des Erlebens und Weltverstehens aus. Wir sind immer schon Verstehende und entwerfen als solche aus strukturgebundenen Möglichkeiten intersubjektive Erfahrungsräume, die für uns mehr und mehrVerbindlichkeit gewinnen, aber auch wieder verlieren. In diesen Sphären stellen sich stets auf's Neue polare Spannungsfelder ein, deren Pole Vertrautes und Fremdes sind. Diese für die Hermeneutik zentrale Spannung ist auch ein wichtiges Thema Gebsers. Das Vertraute ist Gegenstand des ersten Teiles von $U$ \& G (1949), in welchem die über Jahrtausende der Menschheitsgeschichte eingeübten Seinsweisen des Magischen, des Mythischen und des Mentalen beschrieben werden. Sie bilden die "Fundamente der aperspektivischen Welt".

Das (noch) Unvertraute und (vordergründig) Fremde ist hingegen das Integrale, das sich in den "Manifestationen der aperspektivischen Welt« zeigt, die das Thema des zweiten Teiles von $U$ \& G (1953) sind. Vertraut sind uns mentale Erfahrungsräume, bisweilen auch mythische und magische, aber noch nicht der sich immer stärker öffnende integrale Erfahrungsraum. Diese neuartige Sphäre hat auch Rainer Maria Rilke erkundet, dessen Lebenswerk Gebsers persönliche Bewusstwerdung stark befördert hat. Bruno von Flüe hat in seiner phänomenologischen Analyse der "Wirklichkeitserfahrung " Rilkes diesen (integralen) Erfahrungsraum folgendermaßen beschrieben:

"[D]ie neptunisch erlebte Raumhaftigkeit ist ja gerade nicht vordergründige Räumlichkeit, sondern gewahrte Gegenwart des Hintergründigen, Fülle des Offenen, süberzähliges Dasein`, transparent gewordener Raum, ‘Zwischenraum» « ${ }^{11}$

Diese integrale Sphäre, das (vordergründig) Fremde und damit Ungeheure, ist die aperspektivische Welt, die Welt ohne Gegenüber.

\footnotetext{
${ }^{11}$ Bruno von Flüe, Das ganze Gesicht meiner Jahre. Das Geburtsbild Rainer Maria Rilkes. Eine astrologische Deutung, Stuttgart 1988, 87, dort Anm. 6.
} 


\section{III - In Bewegung}

Der immerwährende Wandel des Selbst- und Weltverständnisses in sich wandelnden Erfahrungsräumen ist Gegenstand einer hermeneutischen Topographie des Bewusstseins, für die Gebsers Konzeption der Bewusstseinsstrukturen Erschließungsmöglichkeiten bietet. ${ }^{12}$ Das Strukturgefüge erhellt grundlegende Sinn- und Bedeutungszusammenhänge der im Wandel begriffenen Selbst- und Weltverhältnisse des Menschen. In groben Zügen soll nun der Wandel von Erfahrungsgroßräumen des Magischen, des Mythischen, des Mentalen und des Integralen skizziert werden. Es ist ein hermeneutischer Reisebericht mit Fokus auf die menschheitliche Dimension des Geschehens.

Die magische Bewusstseinsstruktur ist die erste, die sich aus dem Archaischen konstelliert und sich in ihrer grundsätzlichen Ausrichtung durch Weltbezogenheit auszeichnet. Im Magischen, das vom Numinosen bestimmt ist, lebt der Mensch in den Rhythmen und Zyklen der ihn umgreifenden Natur. Die Strukturlogik des Magischen ist das pars pro toto, in welchem der Teil für alles stehen kann und steht. Gebser beschreibt das Magische als

»eine Welt des bloßen, aber sinnreichen Zufalls, nämlich eine Welt, wo alles dem Menschen Zufallende von wirkender Gültigkeit ist, da zu allem und unter allen ein Bezug besteht: das noch nicht zentrierte Ich ist noch über die Welt der Erscheinungen zerstreut. ${ }^{13}$

Durch Rituale und Beschwörungen sucht das magische Bewusstsein auf den Verlauf des Geschehens Einfluss zu nehmen.

Die magische Sphäre erschließt sich dem Menschen durch die fünf Sinne, die Intuition und das Fühlen. Gebser hebt das Ein- und Einsfühlen sowie das Hören als magische Realisationsformen hervor. Er stellt in seiner Phänomenologie des Magischen eine Vielzahl von Bezügen her, die über das Hören Sinn- und Bedeutungszusammenhänge dieser Struktur erschließen. In der Menschheitsgeschichte reicht das Magische tief in die Vor- und Frühgeschichte hinein.

In der Geschichte des Individuums ist das nicht anders. So verweist zum Beispiel Sloterdijk im ersten Band der Sphären auf das fötale

\footnotetext{
${ }^{12}$ Einen Eindruck von dieser hermeneutischen Topographie bietet die synoptische Tafel im Anhang des zweiten Teiles von Ursprung und Gegenwart, die in Längs- und Querschnitten eine Vielzahl von Strukturmerkmalen zusammenfasst und so einen Überblick ermöglicht.

13 Gebser, Ursprung und Gegenwart, Erster Teil, 122.
} 
Hören, das Schweben und Schwingen des Fötus im Fruchtwasser, den akustischen Innenraum der Fruchtblase, und das nachgeburtliche Selbsterlebnis des Neugeborenen beim Gebrauch der eigenen Stimme,

»die als ein vokal-magisches Medium dieVerbindung zur Mutter außerhalb der Leibeshöhle sicherstellt; sie bietet, gleichsam als akustische Nabelschnur, einen Ersatz für die verlorengegangene umbilikale Verbindung. ${ }^{14}$

Wir sind auch später noch, vor allem in der Kindheit, in magischen (und mythischen) Erfahrungsräumen unterwegs. Die Erfahrung der von Gebser betonten magischen Raum- und Zeitlosigkeit (infolge der Ich-Losigkeit), ist uns außerdem beim Meditieren, im Musikhören, das ein "In-der-Musik-Sein" (Sloterdijk) ist, oder auch im Tanzen möglich. Der Tanz ist eine elementare Manifestation der magischen Struktur.

Im Unterschied zur magischen (und auch mentalen) Struktur zeichnet sich die mythische Bewusstseinsstruktur in ihrer grundsätzlichen Ausrichtung durch Selbstbezogenheit aus. Die Seele ist das große Thema des Mythischen, ihre Bewusstwerdung vollzieht sich nach Gebser vornehmlich in Bildern. ${ }^{15}$ Über die Bildung von Mythen schreibt er:

"Mythos: das ist ein Schließen von Mund und Augen; und da es damit ein schweigendes Nach-Innen-Sehen (und ein NachInnen-Hören) ist, ist es ein Ansichtigwerden der Seele, die gesehen, dargestellt, die gehört, hörbar gemacht werden kann. Und Mythos: das ist dies Darstellen, dies Hörbar-Machen; es ist: die Aussage, der Bericht [...] über das Erblickte und Gehörte. Was das eine Mal stummes Bild war, ist das andere Mal tönendes Wort; das Innen-Erschaute und gleichsam Erträumte findet seine polare Entsprechung und Bewußtwerdung in der dichterisch gestalteten Aussage. ${ }^{16}$

So gewinnt der mythische Erfahrungsraum seine Verbindlichkeit für das sich ausbildende Wir-Bewusstsein.

\footnotetext{
${ }^{14}$ Peter Sloterdijk, Blasen. Sphären I, Frankfurt a.M. 1998, 303.

${ }^{15}$ Das ist auch C. G. Jungs tiefenpsychologischer Zugang; siehe: Elmar Schübl, Hermeneutik und Tiefenpsychologie, in: Die Vermessung der Seele. Geltung und Genese der Quantifizierung von Qualia, hg. v. Christian Bachhiesl, Sonja Maria Bachhiesl und Stefan Köchel, Wien 2015, 349-364.

${ }^{16}$ Gebser, Ursprung und Gegenwart, Erster Teil, 147.
} 
Zur Welt kommen - Zur Sprache kommen (1988) lautet der Titel von Sloterdijks Frankfurter Poetik-Vorlesung. Der im Vergleich zum Magischen neuartige mythische Erfahrungsraum verdankt sich der Sprache. Im Mythischen vollzieht sich die Bewusstwerdung der Seele als bildhafte Ausdifferenzierung schöpferischer Innerlichkeit, die zur Sprache gebracht wird; zugleich differenziert sich das Fühlen, als elementarer Zugang zur Welt und zu sich selbst, weiter aus.

Nicht mehr das pars pro toto, sondern Komplementarität ist die Strukturlogik des Mythischen. In der mythischen Sphäre ergänzt sich, vom Mentalen aus betrachtet, Gegensätzliches (Innen-Außen, Subjekt-Objekt etc.) polar, weswegen sie dem mentalen Bewusstsein ambivalent erscheinen muss. Das Sowohl-als-auch ist für das Mythische verbindlich, im Mentalen gilt das Entweder-oder; im Mentalen ist etwas richtig oder falsch, im Mythischen hingegen ist etwas mehr oder weniger stimmig. (Das überlieferte Bild, Homer sei blind gewesen, ist zum Beispiel sehr stimmig.) Das Mythische ist die Sphäre des Traumhaften, des Bildhaften und des Symbolischen. Hier ist die Analogie in ihrem eigentlichen Element: wie oben, so unten wie innen, so außen. Die Bewegtheit der Seele und der Rhythmus der Natur entsprechen sich. Der geschaute innere Kosmos findet seine Entsprechung am nächtlichen Himmel.

$\mathrm{Zu}$ den großen Seelen-Symbolen zählt neben dem Himmel auch das Meer, und die Meerfahrt ist ein Sinnbild für die Erschließung der mythischen Sphäre.

"Nach Durchmessung der eigenen Seele, nach dieser Meerfahrt, die wir als Symbol für eine Bewußtwerdung auffassen, findet der mythische Mensch den andern Menschen, findet er den Partner, vielmehr die ihm persönlich Bestimmte. Auf dem Umweg über das Erwachen zu sich selber erwacht das Du, erwacht im Du die ganze Welt $[\ldots] .1^{17}$

Das vom Menschen erschlossene mentale Ich-Bewusstsein bedeutet intensivierte Reflexivität. Sie wird durch einen Abstand zum Anderen, dem Du, oder, ganz allgemein formuliert, zur Welt gewonnen. Durch diese Distanzierung schafft sich der Mensch den Denkraum und damit die Dimension der Begrifflichkeit und Abstraktion. Die mentale Realisationsform, das Denken, ist raumschaffend und verdinglichend, wodurch sich sofort die ontologische Frage stellt.

Das Mentale ist eine fundamentale Seinsweise des Menschen, die als solche bereits ein Thema des mythischen Bewusstseins ist. Hinrich Knittermeyer bezeichnet in seinen Grundgegebenheiten des

${ }^{17}$ Ebd., 150. 
menschlichen Daseins (1963) den Mythos als die "Geschichte des Sichselbst-Entdeckens des Menschen ${ }^{18}{ }^{18}$ Das In-Erscheinung-Treten des Mentalen ist das Thema des griechischen Mythos von der Geburt der Athene und des jüdisch-christlichen Mythos vom Sündenfall. Es wird in beiden Mythen ein epochales Ereignis erzählt. Das Denken konstituiert das Ich-Bewusstsein, das eine neuartige, eben die mentale Weltsicht eröffnet.

Athene, die Schutzgöttin der Philosophie, entspringt dem durch einen Beilschlag gespaltenen Haupt des Göttervaters Zeus. Das Mentale ist eine Kopfgeburt. Dieser Akt, der die Geburt einer bislang unbekannten Bewusstseinsmöglichkeit beschreibt, erfolgt unter dem großen Staunen aller Götter und einer massiven Erschütterung der den Menschen umgreifenden Natur. Da ist ein unvertrauter und damit noch ungeheurer Erfahrungsraum eröffnet.

Das ist auch die Konsequenz des in der Genesis erzählten Sündenfalls. Adam und Eva essen Früchte vom Baum der Erkenntnis und ignorieren damit ein Gebot Gottes. Das gewonnene Ich-Bewusstsein bedeutet hier die Vertreibung aus dem Paradies, d.h. aus dem Vertrauten der mythischen Sphäre, und den Eintritt in die sich nun eröffnende Geschichte. Erst das Mentale ist auch ein historisches Bewusstsein.

Historiographie und Philosophie treten als Manifestationen der mentalen Struktur praktisch gleichzeitig auf. Das geschah in der griechischen Kultur zuerst in den seit Mitte des 8. Jahrhunderts v. Chr. an den Küsten des Mittelmeeres und des Schwarzen Meeres errichteten Kolonien.

Die Philosophie ist für Gebser jene Manifestation des Mentalen, die am meisten zur Ausgestaltung von intersubjektiven Erfahrungsräumen dieser Bewusstseinsstruktur beigetragen hat. Platon zählt zu den ganz großen Architekten solcher Erfahrungsräume. Vor dem Hintergrund von Gebsers Konzeption lässt sich Platons Philosophieren als eines verstehen, das Momente des Mythischen integriert. Sloterdijk bezeichnet es als eine "Religion des Denkens ${ }^{19}$. Das rationale Moment im platonischen Denken ist die Mathematik. Aber sein Mathematisierungsprogramm umfasst sowohl die Seele und die Natur als auch den sie umgreifenden Kosmos. Die mathematisch

\footnotetext{
${ }^{18}$ Es sind für den Menschen allgemeinverbindliche Prozesse, die in Mythen zur Sprache gebracht werden. Diese Bewusstwerdungsprozesse vollzieht das Individuum im Laufe seines Lebens. Sie geschehen praktisch permanent, wo Menschen ihr Leben gestalten. Mythen sind gewissermaßen Geschichtserzählungen des Unsichtbaren der Weltinnenräume.

${ }^{19}$ Peter Sloterdijk, Philosophische Temperamente.Von Platon bis Foucault, München 2009, 14 .
} 
beschreibbare Ordnung unseres Sonnensystems, die so Rationalität offenbart, entspricht dem von Platon entworfenen Ideen-Kosmos, der zum ersten mentalen Erfahrungsgroßraum werden sollte. Es ist ein Geborgenheit vermittelnder Kosmos.

Das ist bei Aristoteles nicht anders, dessen Rezeption im Hochmittelalter die Hinwendung zum empirisch Fassbaren anzubahnen begann. Der Prozess der Verweltlichung mit seiner im Wandel begriffenen Raumerfahrung erhielt so einen starken Impuls. Gebser verweist auf einen Brief Francesco Petrarcas aus dem Jahr 1336. In dessen Schilderung des - wohl fiktiven - Gipfelerlebnisses auf dem Mont Ventoux komme

"ein erstes Aufleuchten jenes Raumbewußtseins zum Durchbruch, das in der Folge grundlegend die Stellung des europäischen Menschen in und zu der Welt verändert $\aleph^{20}$.

Dieses epochale Ereignis, die »Entdeckung der Landschaft «" ${ }^{21}$, löst bei Petrarca einen Schock aus. Er sucht sofort Halt in der Lektüre der Bekenntnisse und spricht jene Ausführungen an, in welchen Augustinus vor dem Blick auf die Natur warnt, denn damit würden sich die Menschen selbst aufgeben. In Gebsers Worten:

"[D]ie alte Welt, die in dem Worte Augustins, daß die Zeit in der Seele sei, ihre bündigste Formulierung fand, jene alte Welt, in der nichts außerhalb der Seele Liegendes wunderbar und des Anschauens für wert befunden wurde, sie beginnt zu zerbrechen. $\mathbb{1 2}^{22}$

Jacob Burckhardt thematisiert dieses sich stark verändernde mentale Selbst- und Weltverständnis als "Entdeckung der Welt und des Menschen" und als "Entwicklung des Individuums" in seinem Werk Die Kultur der Renaissance in Italien (1860). Es ist dies das Ergebnis eines Perspektivenwechsels. Dazu hat nicht zuletzt auch die Erschließung von Gesetzmäßigkeiten perspektivischer Verfahren beigetragen. Sie sind das Resultat eines objektivierenden Wirklichkeitssinns, der das empirisch Fassbare in den Blick nimmt. Das bedeutet vermessende Erschließung von Räumen der äußeren Welt durch Expeditionen und Aufwertung des Sichtbaren, des Gegenständlichen und damit des Quantifizierbaren in den neuzeitlichen Wissenschaften. Und zugleich bedeutet es auch Erweiterung und Stärkung des Ich-Bewusstseins. Der neuzeitliche Mensch erschafft mit Hilfe der Technik

${ }^{20}$ Gebser, Ursprung und Gegenwart, Erster Teil, 81.

${ }^{21} \mathrm{Ebd}$.

22 Ebd., 85. 
und Wissenschaften die "perspektivische Welt (Gebser) und steht ihr nun tatsächlich gegenüber.

Die kopernikanische Wende, mit der Sprengung des aristotelischen Sphärenmodells, bedeutete einen weiteren Verlust an Geborgenheit in mentalen Erfahrungsräumen. Für dieses Schockerlebnis fand Blaise Pascal folgende Worte: »Das ewige Schweigen der unendlichen Räume versetzt mich in Schrecken. $\mathbb{1}^{23}$

Es bedeutet das Ende der Vorstellung von der Welt als endliches, geschlossenes und hierarchisch geordnetes Ganzes.

Wir sind spätestens seit dem 18. Jahrhundert immer deutlicher in die Offenheit der integralen Sphäre unterwegs. Das ist in vielerlei Hinsicht eine völlig neue Erfahrung und sehr große Herausforderung für die Menschheit. Sloterdijk hat in seiner Sphären-Trilogie herausgearbeitet, dass es bei der Umsiedlung von einer Sphäre in die andere zu Konflikten, Krisen und Katastrophen kommt. Das ist auch Gebsers Befund; aus seiner Sicht tragen dazu defiziente Ausdrucksformen des Bewusstseins bei.

Die integrale Sphäre ist für uns immer noch Neuland, trotz pionierhafter Reisen, die Pfade und Spuren hinterlassen haben. Rainer Maria Rilke zählt zu den Pionieren, die im Integralen unterwegs gewesen sind. Sein »Weltinnenraum« lebt vom Ungegenständlichen und Offenen. Als zentrales Anliegen Rilkes bezeichnet Bruno von Flüe: »die Verwandlung des Sichtbaren, Hiesigen ins Unsichtbare unserer fühlenden Herzräume « ${ }^{24}$.

Wir werden bei unseren Erkundungen des integralen Erfahrungsraumes immer weiter und tiefer vorstoßen, im Bewusstsein des Unterwegs-Seins und im Unterwegssein, für immer.

- PD Dr. Elmar Schübl, promovierter Philosoph und habilitierter Wissenschaftshistoriker, lehrt an der Universität Graz. Er ist Mitherausgeber der auf fünf Bände konzipierten Jean-Gebser-Reihe (JGR), die im Verlag Chronos (Zürich) erscheint.

${ }^{23}$ Zitiert nach: Safranski, Selbstverhältnisse, Weltverhältnisse, 77.

${ }^{24}$ Flüe, Das ganze Gesicht meiner Jahre, 91. 